\section{Thermoelectric Materials, \\ Phenomena, and Applications: A Bird's Eye View}

\section{Terry M. Tritt and M.A. Subramanian, Guest Editors}

\begin{abstract}
High-efficiency thermoelectric (TE) materials are important for power-generation devices that are designed to convert waste heat into electrical energy. They can also be used in solid-state refrigeration devices. The conversion of waste heat into electrical energy may play an important role in our current challenge to develop alternative energy technologies to reduce our dependence on fossil fuels and reduce greenhouse gas emissions.

An overview of various TE phenomena and materials is provided in this issue of MRS Bulletin. Several of the current applications and key parameters are defined and discussed. Novel applications of TE materials include biothermal batteries to power heart pacemakers, enhanced performance of optoelectronics coupled with solid-state TE cooling, and power generation for deep-space probes via radioisotope TE generators. A number of different systems of potential TE materials are currently under investigation by various research groups around the world, and many of these materials are reviewed in the articles in this issue. These range from thin-film superlattice materials to large singlecrystal or polycrystalline bulk materials, and from semiconductors and semimetals to ceramic oxides. The phonon-glass/electron-crystal approach to new TE materials is presented, along with the role of solid-state crystal chemistry. Research criteria for developing new materials are highlighted.
\end{abstract}

Keywords: energy, thermal conductivity, thermoelectricity.

\section{Introduction}

\section{Thermoelectric Phenomena:} Background and Applications

Over the past decade, there has been heightened interest in the field of thermoelectrics, driven by the need for more efficient materials for electronic refrigeration and power generation. ${ }^{1,2}$ Some of the research efforts focus on minimizing the lattice thermal conductivity, while other efforts focus on materials that exhibit large power factors. Proposed industrial and military applications of thermoelectric
(TE) materials are generating increased activity in this field by demanding higherperformance high-temperature TE materials than those that are currently in use. The demand for alternative energy technologies to reduce our dependence on fossil fuels leads to important regimes of research, including that of hightemperature energy harvesting via the direct recovery of waste heat and its conversion into useful electrical energy. Thus, the development of higher-performance
TE materials is becoming ever more important. Power-generation applications are currently being investigated by the automotive industry as a means to develop electrical power from waste engine heat from the radiator and exhaust systems for use in next-generation vehicles. In addition, TE refrigeration applications include seat coolers for comfort and electronic component cooling. Of course, the deepspace applications of NASA's Voyager and Cassini missions using radioisotope thermoelectric generators (RTGs) are well established (see Reference 3 and the article by Yang and Caillat in this issue). A key factor in developing these technologies is the development of higher-performance TE materials, either completely new materials or through more ingenious materials engineering of existing materials.

Thermoelectric refrigeration is an environmentally "green" method of smallscale, localized cooling in computers, infrared detectors, electronics, and optoelectronics as well as many other applications. However, most of the electronics and optoelectronics technologies typically require only small-scale or localized spot cooling of small components that do not impose a large heat load. If significant economical cooling can be achieved, the resulting "cold computing" could produce speed gains of $30-200 \%$ in some computer processors based on complementary metal oxide semiconductor (CMOS) technology. Cooling of the processors is perceived by many to be the fundamental limit to electronic system performance. ${ }^{4}$ Thus, the potential payoff for the development of low-temperature TE refrigeration devices is great, and the requirement for compounds with properties optimized over wide temperature ranges has led to a much expanded interest in new TE materials. Recent utilization of Peltier coolers (see next section) for the refrigeration of biological specimens and samples is an emerging TE application.

The development and potential of bulk materials for TE applications is an active area of research. High-temperature bulk materials such as skutterudites, clathrates, half-Heusler alloys, and complex chalcogenides are being investigated (see the article by Nolas et al. in this issue). These materials possess complex crystal structures and exhibit properties that are favorable for potential thermoelectric materials. For example, skutterudites and clathrates are cage-like materials that have voids in which "rattler" atoms are inserted to significantly lower the thermal conductivity due to the rattling atoms' ability to scatter phonons. Recently, ceramic oxide materials have also shown potential as high- 
temperature TE materials (see Koumoto et al. in this issue). The potential of nanomaterials and their role in TE research are an emerging area of interest (see Rao et al. in this issue). Bulk material applications are demanding new breakthroughs in both materials and device engineering (see Yang and Caillat in this issue). The role of thin-film properties, applications, and recent results is also very important (see Böttner et al. in this issue). A more complete overview of state-of-theart materials, a theoretical and experimental discussion of the basic principles, and an overview of some of the recent developments and materials are given in texts by Tritt $^{2}$ and Nolas. ${ }^{5}$

\section{Seebeck and Peltier Effects}

A discussion of thermoelectric effects and devices should start with one of the most fundamental TE phenomena, the Seebeck effect, or thermopower. ${ }^{6-8}$ In the early 1800s, Seebeck observed that when two dissimilar materials are joined together and the junctions are held at different temperatures ( $T$ and $T+\Delta T$ ), a voltage difference $(\Delta V)$ develops that is proportional to the temperature difference $(\Delta T){ }^{6}$ The ratio of the voltage developed to the temperature gradient $(\Delta V / \Delta T)$ is related to an intrinsic property of the materials called the Seebeck coefficient, $\alpha$. The Seebeck coefficient is very low for metals (only a few $\mu \mathrm{V} / \mathrm{K}$ ) and much larger for semiconductors (typically a few hundred $\mu \mathrm{V} / \mathrm{K}) .{ }^{9}$ A related effect-the Peltier effect-was discovered a few years later by Peltier, ${ }^{10}$ who observed that when an electrical current is passed through the junction of two dissimilar materials, heat is either absorbed or rejected at the junction, depending on the direction of the current. This effect is due largely to the difference in Fermi energies of the two materials. These two effects are related to each other, as shown in the definition of the Peltier coefficient, $\Pi=\alpha T$. The rate at which the Peltier heat is liberated or rejected at the junction $\left(Q_{\mathrm{P}}\right)$ is given by $Q_{\mathrm{P}}=$ $\alpha I T$, where $I$ is the current through the junction and $T$ is the temperature in kelvin. There are also a number of thermomagnetic effects such as the Hall, Ettingshausen, and Nernst effects that are beyond the scope of this article. The reader is referred to the text by Nolas et $a .^{5}$ for a discussion of these effects.

\section{Definition and Description of the Figure of Merit and Thermoelectric Performance}

The potential of a material for TE applications is determined in large part by a measure of the material's figure of merit, ZT:*

$$
Z T=\frac{\alpha^{2} \sigma T}{\kappa}=\frac{\alpha^{2} T}{\rho \kappa}
$$

where $\alpha$ is the Seebeck coefficient, $\sigma$ is the electrical conductivity, $\rho$ is the electrical resistivity, and $\kappa$ is the total thermal conductivity $\left(\kappa=\kappa_{\mathrm{L}}+\kappa_{\mathrm{E}}\right.$, the lattice and electronic contributions, respectively). The power factor, $\alpha^{2} \sigma T$ (or $\alpha^{2} T / \rho$ ), is typically optimized in narrow-gap semiconducting materials as a function of carrier concentration (typically $\sim 10^{19}$ carriers $/ \mathrm{cm}^{3}$ ), through doping, to give the largest ZT. ${ }^{9}$ High-mobility carriers are most desirable, in order to have the highest electrical conductivity for a given carrier concentration. The ZT for a single material is somewhat meaningless, since an array of TE couples is utilized in a device or module.

There are two materials in the TE couple, which is shown in Figure 1, an n-type and a $p$-type. Ignoring parasitic contributions that reduce the device performance, such as contact resistance and radiation effects, the resulting figure of merit for the couple (based solely on the TE materials) is given by

$$
Z T=\frac{\left(\alpha_{p}-\alpha_{n}\right)^{2} T}{\left[\left(\rho_{n} \kappa_{n}\right)^{1 / 2}+\left(\rho_{p} \kappa_{p}\right)^{1 / 2}\right]} .
$$

The coefficient of performance $\phi$ (refrigeration mode) and the efficiency $\eta$ (powergeneration mode) of the TE couple are directly related to the figure of merit shown in Equation 3 for the efficiency. The efficiency $(\eta)$ of the TE couple is given by the power input to the load $(W)$ over the net heat flow rate $\left(Q_{\mathrm{H}}\right)$, where $Q_{\mathrm{H}}$ is positive for heat flow from the source to the sink:

$$
\begin{aligned}
& \eta=\frac{W}{Q_{\mathrm{H}}} \\
& =\frac{T_{\mathrm{H}}-T_{\mathrm{C}}}{T_{\mathrm{H}}}\left[\frac{\left(1+Z T_{\mathrm{M}}\right)^{1 / 2}-1}{\left(1+Z T_{\mathrm{M}}\right)^{1 / 2}+\left(T_{\mathrm{C}} / T_{\mathrm{H}}\right)}\right],
\end{aligned}
$$

where $T_{\mathrm{H}}$ is the hot-side temperature, $T_{\mathrm{C}}$ is the cold-side temperature, and $T_{\mathrm{M}}$ is the average temperature. Thus, one can see

*The expressions for figure of merit, $\mathrm{Z}$ and $\mathrm{ZT}$, are used interchangeably in the field of thermoelectrics. $Z$ is the figure of merit with units of $1 / \mathrm{K}(1 / T)$, and $Z T$ is the dimensionless (unitless) figure of merit. Both must specify the temperature at which the quoted value was obtained. that $\eta$ is proportional to $\left(1+Z T_{M}\right)^{1 / 2}$ and that the efficiency would approach the Carnot efficiency if ZT were to approach infinity.

\section{Thermoelectric Modules: Devices}

The Peltier effect is the basis for many modern-day TE refrigeration devices, and the Seebeck effect is the basis for TE power-generation devices. The versatility of TE materials is illustrated in Figure 1, which shows a TE couple composed of an $n$-type (negative thermopower and electron carriers) and a $p$-type (positive thermopower and hole carriers) semiconductor material connected through metallic electrical contact pads. Both refrigeration and power generation may be accomplished using the same module, as shown in Figure 1. A TE module or device is built up of an array of these couples, arranged electrically in series and thermally in parallel. Thermoelectric energy conversion utilizes the Seebeck effect, wherein a temperature gradient is imposed across the device, resulting in a voltage that can be used to drive a current through a load resistance or device. This is the direct conversion of heat into electricity. Conversely, the Peltier heat generated when an electric current is passed through a TE material provides a temperature gradient, with heat being absorbed on the cold side, transferred through (or pumped by) the TE materials, and rejected at the sink, thus providing a refrigeration capability. The advantages of TE solid-state energy conversion are compactness, quietness (no moving parts), and localized heating or cooling. In addition, energy in the form of waste heat ( $0 \%$ efficiency) that would normally be lost may be converted into useful electrical energy ( $\geq 7-8 \%$ efficiency) using a TE power-generation device.

The best TE materials currently used in devices have $Z T \approx 1$. This value has been a practical upper limit for more than 30 years, yet there are no theoretical or thermodynamic reasons for $\mathrm{ZT} \approx 1$ as an upper barrier. As seen from Equation 1, ZT may be increased by decreasing $\kappa_{\mathrm{L}}$ or by increasing either $\alpha$ or $\sigma$. However, $\sigma$ is tied to the electronic thermal conductivity, $\kappa_{\mathrm{E}}$, through the Wiedemann-Franz relationship, and the ratio is essentially constant at a given temperature.

Some of the goals of current research efforts are to find new materials that either raise the current efficiency of TE devices (i.e., increase ZT) or have the capability of operating in new and broader temperature regimes, especially at lower temperatures $(T<250 \mathrm{~K})$ and higher temperatures $(T>400 \mathrm{~K})$. Over the past 30 years, 


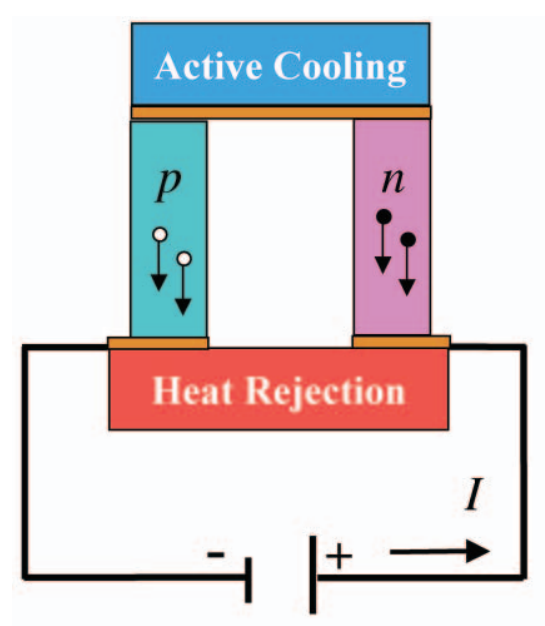

\section{Refrigeration Mode}

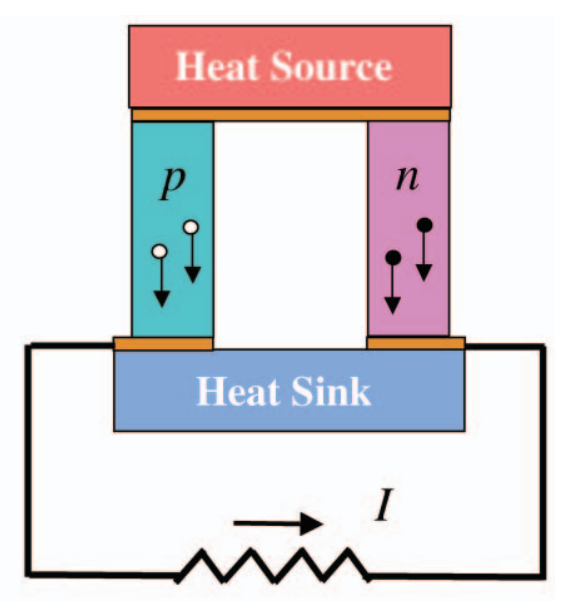

\section{Power-Generation Mode}

Figure 1. Diagram of a Peltier thermoelectric couple made of an n-type and a p-type thermoelectric material. Refrigeration or power-generation modes are possible, depending on the configuration. I is current.

alloys based on the $\mathrm{Bi}_{2} \mathrm{Te}_{3}$ system $\left[\left(\mathrm{Bi}_{1-x} \mathrm{Sb}_{x}\right)_{2}\left(\mathrm{Te}_{1-x} \mathrm{Se}_{x}\right)_{3}\right]$ and the $\mathrm{Si}_{1-y} \mathrm{Ge}_{y}$ system have been extensively studied and optimized for their use as TE materials to perform in a variety of solid-state TE refrigeration and power-generation applications. $^{11,12}$ These traditional TE materials have undergone extensive investigation, and there appears to be little room for future improvement in the common bulk structures. However, recent results on nanostructures of traditional TE materials have shown a promising new direction for these materials. In addition, entirely new classes of compounds will have to be investigated. Figure 2 shows $Z T$ as a function of temperature for the $\mathrm{Bi}_{2} \mathrm{Te}_{3}$ and $\mathrm{Si}_{1-y} \mathrm{Ge}_{y}$ materials as well as many of the more recent bulk materials that have been developed over the last decade. The ZT of more exotic structures such as superlattices and quantum dot structures are not shown here but are addressed in the article by Böttner et al. in this issue.

\section{Transport Properties}

The thermopower, or Seebeck coefficient, can be thought of as the heat per carrier over temperature or, more simply, the entropy per carrier, $\alpha \approx C / q$, where $C$ is the specific heat and $q$ is the charge of the carrier. ${ }^{7}$ For the case of a classical gas, each particle has an energy of $3 / 2\left(k_{\mathrm{B}} T\right)$, where $k_{\mathrm{B}}$ is the Boltzmann constant. The thermopower is thus approximately $k_{\mathrm{B}} / e$, where $e$ is the charge of the electron. For metals, the heat per carrier is essentially a product of the electronic specific heat and the temperature divided by the number of carriers $(N)$, that is, $\alpha \approx C_{\mathrm{el}} T / N$, and then $\alpha$ is approximately

$$
\alpha \approx \frac{C_{\mathrm{el}}}{q} \approx\left(\frac{k_{\mathrm{B}}}{e}\right) \frac{k_{\mathrm{B}} T}{E_{\mathrm{F}}},
$$

where $E_{\mathrm{F}}$ is the Fermi energy (related to the chemical potential of the material).
The Fermi energy is basically the energy such that at $T \approx 0$, all the states above this energy are vacant and all the states below are occupied. The quantity $k_{\mathrm{B}} / e \approx 87 \mu \mathrm{V} / \mathrm{K}$ is a constant that represents the thermopower of a classical electron gas. Metals have thermopower values of much less than $87 \mu \mathrm{V} / \mathrm{K}$ (on the order of $1-10 \mu \mathrm{V} / \mathrm{K}$ ) and decrease with decreasing temperature, that is, $E_{\mathrm{F}} \gg k_{\mathrm{B}} T$ ).

In a semiconductor, a charged particle must first be excited across an energy gap $E_{\mathrm{g}}$. In this case, the thermopower is approximated by

$$
\alpha \approx \frac{C_{\mathrm{el}}}{q} \approx\left(\frac{k_{\mathrm{B}}}{e}\right) \frac{E_{\mathrm{g}}}{k_{\mathrm{B}} T} .
$$

Thus, the thermopower is larger than the characteristic value of $87 \mu \mathrm{V} / \mathrm{K}$ and increases with decreasing temperature. Semiconductors can exhibit either electron conduction (negative thermopower) or hole conduction (positive thermopower). The thermopower for different carrier types is given by a weighted average of their electrical conductivity values $\left(\sigma_{n}\right.$ and $\left.\sigma_{p}\right)$ :

$$
\alpha \approx \frac{\left(\alpha_{n} \sigma_{n}+\alpha_{p} \sigma_{p}\right)}{\left(\sigma_{n}+\sigma_{p}\right)}
$$

It is necessary to dope the semiconductors with either donor or acceptor states to

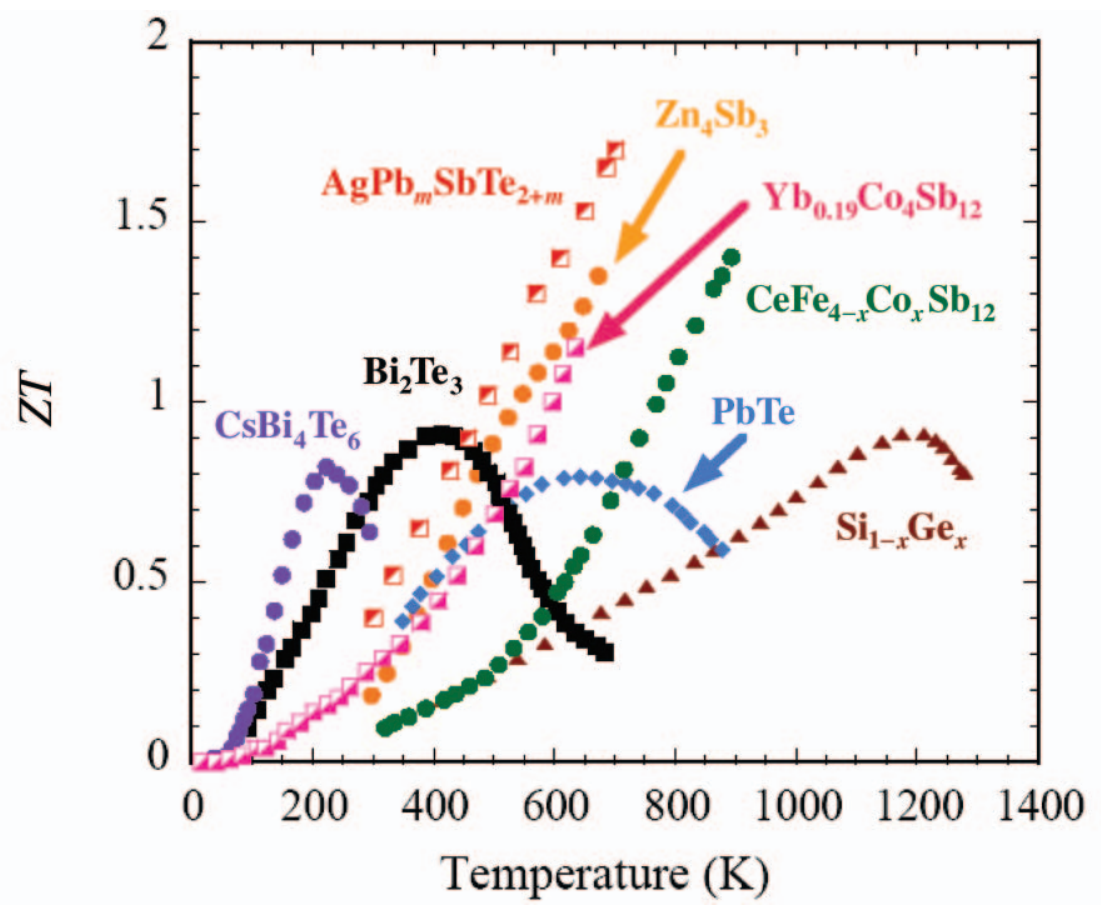

Figure 2. Figure of merit ZT shown as a function of temperature for several bulk thermoelectric materials. 
allow extrinsic conduction of the appropriate carrier type, electrons or holes, respectively. It is apparent that the total thermopower will be lower than that of either of the individual contributions, unless the direct bandgap is large enoughtypically on the order of $10\left(k_{\mathrm{B}} T\right)$ - to effectively minimize minority carrier contributions. Typical thermopower values required for good TE performance are on the order of $150-250 \mu \mathrm{V} / \mathrm{K}$ or greater.

For high-temperature applications, it is important to minimize the contribution of minority carriers in order to maintain a high thermopower. In addition, the thermal stability of the materials is an essential aspect. Atomic diffusion within the materials and interdiffusion of contacts can seriously deteriorate the properties of a given material at high temperatures. Aspects of this are discussed elsewhere. ${ }^{2,5}$ These materials and devices are expected to operate at elevated temperatures for long periods of time without deterioration of their properties or performance. The effects of diffusion and thermal annealing are important to thoroughly investigate and understand in any set of potential TE materials over the expected operating temperature range of the materials.

The description of electrical conductivity for metals and semiconductors has been covered extensively in many texts on solid-state physics, and the reader is referred there. ${ }^{13}$ There are a significant number of carriers and states available for conduction in metals, typically $n \approx 10^{22}$ carriers $/ \mathrm{cm}^{3}$. The electrical conductivity is then very large for metals, on the order of $10^{6}(\Omega \mathrm{cm})^{-1}$. Again, for semiconductors, the carriers must be thermally excited across a gap for conduction to occur, as shown from the activated behavior that is derived for the temperature-dependence of the electrical conductivity $[\sigma \approx$ $\left.\sigma_{0} \exp \left(-E_{\mathrm{g}} / k_{\mathrm{B}} T\right)\right]$. There are two primary ways to achieve a high conductivity in a semiconductor, either by having a very small gap to excite across $\left(E_{\mathrm{g}} / k_{\mathrm{B}} T\right)$ or by having very high-mobility carriers, as discussed later. Typical values of the electrical conductivity for a good TE material are on the order of about $10^{3}(\Omega \mathrm{cm})^{-1}$.

The thermal conductivity $\kappa$ is related to the transfer of heat through a material, either by the electrons or by quantized vibrations of the lattice, called phonons, such that $\kappa=\kappa_{\mathrm{L}}+\kappa_{\mathrm{E}}$, as mentioned earlier. The electrical conductivity and the thermal conductivity are interrelated, in that $\sigma$ is tied to $\kappa_{\mathrm{E}}$ through the WiedemannFranz relationship: $\kappa_{\mathrm{E}}=L_{0} \sigma T$, where the Lorentz number $L_{0}=2.45 \times 10^{-8} \mathrm{~W} \Omega / \mathrm{K}^{2}$ [or $L_{0}=2.45 \times 10^{-8}\left(\mathrm{~V}^{2} / \mathrm{K}^{2}\right)$ ]. The lattice thermal conductivity is discussed later in this article, in the section on minimum thermal conductivity. Typical thermal conductivity values for a good TE material are $\kappa<2 \mathrm{~W} \mathrm{~m}^{-1} \mathrm{~K}^{-1}$, and typically, $\kappa_{\mathrm{L}} \approx \kappa_{\mathrm{E}}$.

\section{Investigating New Thermoelectric Materials \\ The "Phonon-Glass/Electron- Crystal" Approach}

Slack has described the chemical characteristics of candidates for a good TE material. ${ }^{14} \mathrm{He}$ states that the candidates should be narrow-bandgap semiconductors with high-mobility carriers. Mahan has also described the characteristics of good TE materials, ${ }^{15,16}$ agreeing with Slack that the candidate material is typically a narrow-bandgap semiconductor $\left[E_{\mathrm{g}} \approx\right.$ $10\left(k_{\mathrm{B}} T\right)$, or $0.25 \mathrm{eV}$ at $\left.300 \mathrm{~K}\right]$. Also, the mobility of the carriers must remain high $\left(\mu \approx 2000 \mathrm{~cm}^{2} / \mathrm{V} \mathrm{s}\right.$ ), while the lattice thermal conductivity must be minimized. In semiconductors, the Seebeck coefficient and electrical conductivity (both in the numerator of ZT) are strong functions of the doping level and chemical composition. These quantities must therefore be optimized for good TE performance. The thermal conductivity of complex materials can often be modified by chemical substitutions, and the lattice thermal conductivity needs to be as low as possible. Understanding these various effects and selecting optimization strategies can be an exceedingly difficult problem, because in complex materials there are often many possible degrees of freedom. Slack suggested that the best TE material would behave as a "phonon-glass/electron-crystal" (PGEC); that is, it would have the electrical properties of a crystalline material and the thermal properties of an amorphous or glass-like material. Materials engineering and the crystal chemistry approach to good TE materials are discussed later.

\section{Minimum Thermal Conductivity}

In many areas of research related to new TE materials, attempts are being made to reduce the lattice part of the thermal conductivity to essentially its minimum value, that is, where a minimum lattice thermal conductivity is achieved (when all the phonons have a mean free path essentially equal to the interatomic spacing of the constituent atoms). This is being attempted by scattering phonons in different frequency ranges using a variety of methods such as mass fluctuation scattering (a mixed crystal, in ternary and quaternary compounds), "rattling" scattering, grain-boundary scattering (due to the size of the grains), and interface scattering in thin films or multilayer systems.
The lattice thermal conductivity is given by $\kappa_{\mathrm{L}} \approx(1 / 3)\left(v_{\mathrm{s}} C L_{\mathrm{ph}}\right)$, where $v_{\mathrm{s}}$ is the velocity of sound, $C$ is the heat capacity, and $L_{\mathrm{ph}}$ is the mean free path of the phonons. At high temperatures $(T>\sim 300 \mathrm{~K})$, the sound velocity and the heat capacity are essentially temperature-independent in typical materials. Therefore, the magnitude and the temperature-dependence of $\kappa_{\mathrm{L}}$ are basically determined by the mean free path of the phonons. Slack defined the minimum thermal conductivity $\left(\kappa_{\min }\right)$ as the thermal conductivity when the mean free path is essentially limited by the interatomic distance between the atoms within the crystal. ${ }^{17}$ Typical analysis of $\kappa_{\min }$ results in values of $\kappa_{\text {min }} \approx 0.25-0.5 \mathrm{~W} \mathrm{~m}^{-1} \mathrm{~K}^{-1}$. 14,17

\section{Minimum Thermopower}

There are certain practical limits for each of the parameters used to calculate ZT. These practical limits must be possible in order to achieve a material viable for thermoelectric applications. For example, in $\mathrm{Bi}_{2} \mathrm{Te}_{3}$, in order to achieve a $\mathrm{ZT} \approx 1$ at $T$ $=320 \mathrm{~K}, \sigma \approx 1 \mathrm{~m} \Omega \mathrm{cm}, \alpha \approx 225 \mu \mathrm{V} / \mathrm{K}$, and $\kappa \approx 1.5 \mathrm{~W} \mathrm{~m}^{-1} \mathrm{~K}^{-1}$. We have already discussed the ZT "barrier," which in effect is given by minimizing the thermal conductivity. It is practical to investigate materials where the electronic and lattice terms are comparable, on the order of 0.75-1 $\mathrm{W} \mathrm{m}^{-1} \mathrm{~K}^{-1}$. Let us look at the hypothetical situation of a material in which the lattice thermal conductivity is zero $\left(\kappa_{\mathrm{L}}=0\right)$. We will also assume the scattering in this system is elastic and that the Wiedemann-Franz relationship, slightly rearranged $\left[\kappa_{\mathrm{E}} / \sigma=L_{0} T\right]$, is well behaved in this material. Then we can rewrite Equation 1 as

$$
\mathrm{ZT}=\alpha^{2} T / \rho \kappa_{\mathrm{E}}=\alpha^{2} / L_{0} .
$$

Therefore, for a material to be a viable TE material, it must possess a minimum thermopower that is directly related to the value of $Z T$ and $L_{0}$. Given this description, in order to achieve a certain value of $Z T$, the material would require that $\alpha=\left(L_{0}\right)^{0.5}$ $=157 \mu \mathrm{V} / \mathrm{K}$ for $Z T=1$, and $\alpha=\left(2 L_{0}\right)^{0.5}=$ $225 \mu \mathrm{V} / \mathrm{K}$ for $Z T=2$. Of course, any "real" material will possess a finite $\kappa_{\mathrm{L}}$, and these values for the thermopower will have to be higher to achieve the projected values of $Z T$.

\section{Solid-State Crystal Chemistry Approaches to Advanced Thermoelectric Materials}

Thermoelectrics has always been a materials design problem involving intricate tuning of structure-property relationships in complex solids through principles of solid-state chemistry and physics. The dis- 
cussion thus far indicates that new materials must be able to eventually achieve certain minimum values of important parameters in order to be considered as a potential TE material. It does not matter if a material has a $\kappa_{\mathrm{L}} \approx \kappa_{\min }$; if it cannot be "tuned" or doped in order to attain a minimum thermopower $(150 \mu \mathrm{V} / \mathrm{K})$, it will not be able to achieve $Z T \approx 1$.

\section{Classical Approach: Bulk Binary Semiconductors}

Within the framework of simple electronic band structure of solids, in general, metals are poor TE materials. Hence, most of the early TE work put much emphasis on semiconductors. ${ }^{18}$ As stated earlier, in order to have a maximum ratio of electrical to thermal conductivity, the material should have a low carrier concentration, on the order of $10^{18}-10^{19} \mathrm{~cm}^{3}$, with very high mobilities. Crystal structure and bonding strongly influence the mobility. Materials with diamond or zinc-blende structures with a high degree of covalent bonding frequently have high mobilities (e.g., Si, Ge, InSb), but also exhibit high thermal conductivity values. On the other hand, low lattice thermal conductivities are found in conjunction with low Debye temperatures and high anharmonic lattice vibrations. These conditions are best satisfied by highly covalent intermetallic compounds and alloys of the heavy elements such as $\mathrm{Pb}, \mathrm{Hg}, \mathrm{Bi}, \mathrm{Tl}$, or $\mathrm{Sb}$, and $\mathrm{S}, \mathrm{Se}$, or $\mathrm{Te}$. Once a material system has been selected with a favorable electrical-to-thermal conductivity ratio, one optimizes the composition to further enhance the ZT by doping the material to maximize the density of states at the Fermi level and achieve a high Seebeck coefficient.

The most studied TE material, $\mathrm{Bi}_{2} \mathrm{Te}_{3}$, crystallizes in a layer structure (Figure 3) with rhombohedral-hexagonal symmetry with space group $R \overline{3} m\left(D_{3 d}^{5}\right)$. The hexagonal unit cell dimensions at room temperature are $a=3.8 \AA$ and $c=30.5 \AA$. The layers stacked along the $c$-axis are

$\cdots \mathrm{Te}-\mathrm{Bi}-\mathrm{Te}-\mathrm{Bi}-\mathrm{Te} \cdots \mathrm{Te}-\mathrm{Bi}-\mathrm{Te}-\mathrm{Bi}-\mathrm{Te} \cdots$.

The $\mathrm{Bi}$ and Te layers are held together by strong covalent bonds, whereas the bonding between adjacent Te layers is of the van der Waals type. This weak binding between the Te layers accounts for the ease of cleavage along the plane perpendicular to the $c$-axis and the anisotropic thermal and electrical transport properties of $\mathrm{Bi}_{2} \mathrm{Te}_{3}$. For example, the thermal conductivity along the plane perpendicular to the $c$-axis $\left(1.5 \mathrm{~W} \mathrm{~m}^{-1} \mathrm{~K}^{-1}\right)$ is nearly twice that of the value along the $c$-axis direction $(0.7 \mathrm{~W}$
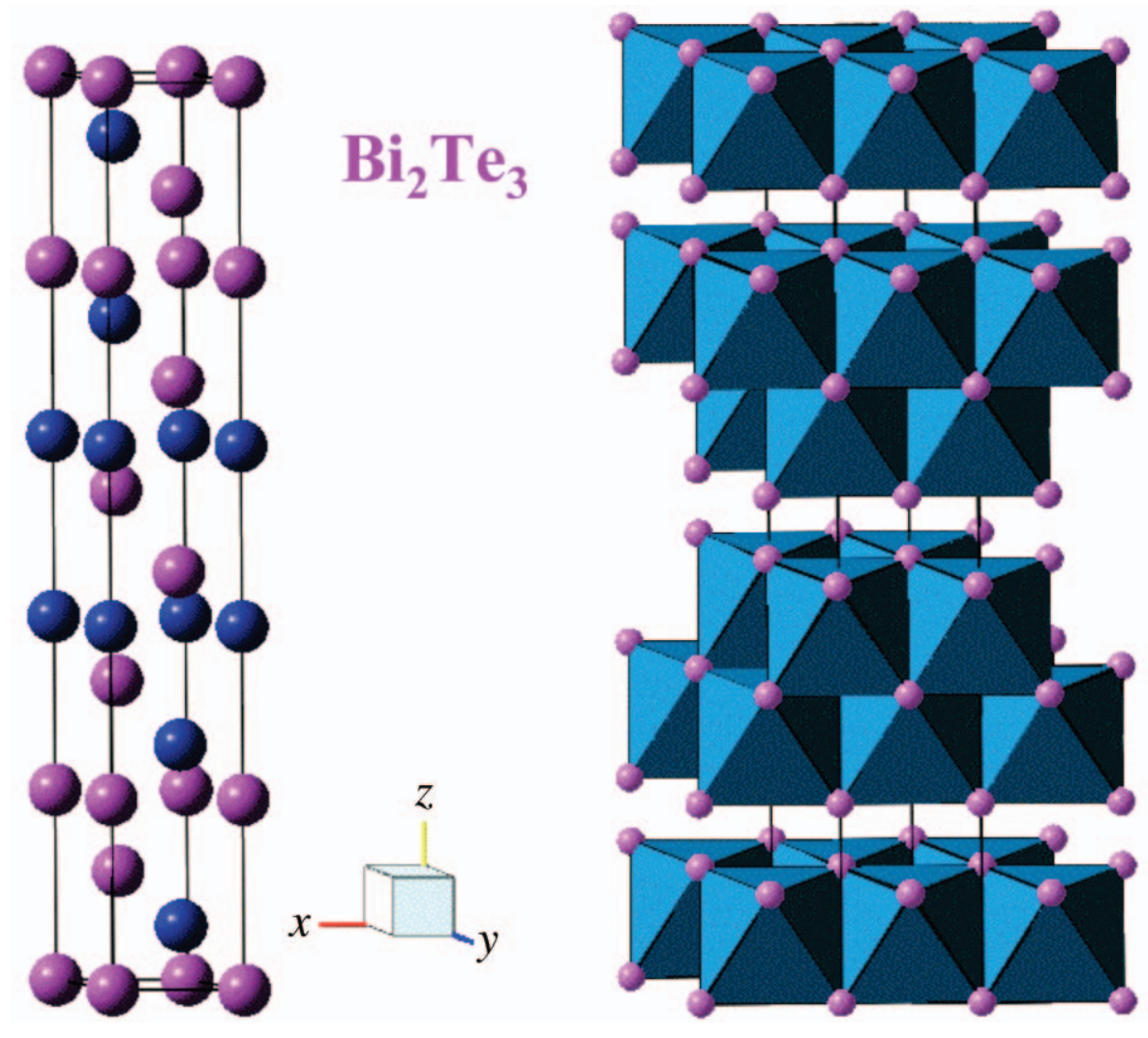

Figure 3. Crystal structure of the state-of-the-art thermoelectric material, $\mathrm{Bi}_{2} \mathrm{Te}_{3}$. The blue atoms are Bi and the pink atoms are Te.

$\left.\mathrm{m}^{-1} \mathrm{~K}^{-1}\right)$. When grown from a melt or by zone refining, the $\mathrm{Bi}_{2} \mathrm{Te}_{3}$ crystals are always nonstoichiometric and show $p$-type behavior. On the other hand, $n$-type materials could be grown from the melt containing excess Te, iodine, or bromine. The thermal conductivity values of both $p$ - and $n$-type $\mathrm{Bi}_{2} \mathrm{Te}_{3}$ are $\sim 1.9 \mathrm{~W} \mathrm{~m}^{-1} \mathrm{~K}^{-1}$, giving a $\mathrm{ZT}$ of about 0.6 near room temperature. Ioffe $^{9}$ suggested that alloying could further reduce the lattice thermal conductivity of $\mathrm{Bi}_{2} \mathrm{Te}_{3}$ through the scattering of short-wavelength acoustic phonons. The optimum compositions for thermoelectric cooling devices are normally $\mathrm{Bi}_{2} \mathrm{Te}_{2.7} \mathrm{Se}_{0.3}$ (n-type) and $\mathrm{Bi}_{0.5} \mathrm{Sb}_{1.5} \mathrm{Te}_{3}$ (p-type) with $\mathrm{ZT} \approx 1$ near room temperature.

In contrast to $\mathrm{Bi}_{2} \mathrm{Te}_{3}, \mathrm{PbTe}$ crystallizes in a cubic $\mathrm{NaCl}$-type crystal structure, and the TE properties are isotropic. Both $p$ type and $n$-type thermoelements can be produced by doping of acceptors (e.g., $\mathrm{Na}_{2} \mathrm{Te}$ or $\mathrm{K}_{2} \mathrm{Te}$ ) or donors $\left(\mathrm{PbI}_{2}, \mathrm{PbBr}_{2}\right.$, or $\mathrm{Ge}_{2} \mathrm{Te}_{3}$ ). In analogy with the $\mathrm{Bi}_{2} \mathrm{Te}_{3}$, the solid-solution compositions (e.g., PbTe$\mathrm{SnTe}$ ) have been made to lower the lattice thermal conductivity. ${ }^{19}$ The $Z T$ value of $\mathrm{PbTe}$ solid solutions is low near room temperature but rises to $\mathrm{ZT} \approx 0.7$ at $700 \mathrm{~K}$, making PbTe a prime candidate for power generation in that temperature range. It is possible to achieve $Z T$ in excess of unity at $700 \mathrm{~K}$ in structurally related solidsolution compositions, $\mathrm{AgSbTe}_{2}(80 \%)$ $\mathrm{GeTe}(20 \%)$, known as TAGS (alloys containing $\mathrm{Te}, \mathrm{Ag}$, Ge, Sb). However, due to high-temperature stability issues, these compositions are not currently favored in TE devices.

Neither Si nor Ge is a good TE material, as the lattice thermal conductivity is very large $\left(150 \mathrm{~W} \mathrm{~m}^{-1} \mathrm{~K}^{-1}\right.$ for $\mathrm{Si}$ and $63 \mathrm{~W} \mathrm{~m}^{-1}$ $\mathrm{K}^{-1}$ for $\mathrm{Ge}$ ). The lattice thermal conductivity can be substantially reduced by alloy formation between the two elements. The best alloy composition is $\mathrm{Si}_{0.7} \mathrm{Ge}_{0.3}$; its thermal conductivity is about $10 \mathrm{~W} \mathrm{~m}^{-1} \mathrm{~K}^{-1}$, and the reduction relative to $\mathrm{Si}$ and $\mathrm{Ge}$ is apparently due to the increased phonon-phonon and phonon-electron scattering. ${ }^{19}$ Remarkably, such a large reduction does not unduly reduce the carrier mobility, and $Z T \approx 0.6-0.7$ could be realized at elevated temperatures. Due to their exceptional stability at high temperatures $(\sim 1200 \mathrm{~K})$, these alloys are of interest to NASA for use in RTGs in deep-space probes. 


\section{Modern Solid-State Chemistry Design Concepts for High-ZT Materials \\ Complex Inorganic Structures. Most of} the earlier investigations mentioned so far focused on binary intermetallic semiconductor systems. Recent approaches to high-performance bulk TE materials focus on ternary and quaternary chalcogenides containing heavy atoms with lowdimensional or isotropic complex structures to take advantage of the large carrier effective masses and low lattice thermal conductivity associated with such systems. ${ }^{20}$ Along these lines, $\mathrm{CsBi}_{4} \mathrm{Te}_{6}$ possessing the layered structure has been identified as a material showing a ZT of 0.8 at $225 \mathrm{~K}$, which is $40 \%$ greater than that of the $\mathrm{Bi}_{2-x} \mathrm{Sb}_{x} \mathrm{Te}_{3-y} \mathrm{Se}_{y}$ alloys. ${ }^{21}$ Other potential low-temperature TE materials currently under investigation are lowdimensional semiconducting or semimetallic doped layered pentatellurides $\left(\mathrm{ZrTe}_{5}\right.$ and $\left.\mathrm{HfTe}_{5}\right)$. ${ }^{22}$ These compounds have a structure similar to $\mathrm{Bi}_{2} \mathrm{Te}_{3}$, with van der Waals gaps between the individual layers. Although doped pentatellurides exhibit very high power factors (exceeding the optimally doped $\mathrm{Bi}_{2} \mathrm{Te}_{3}$ solid solutions) in the low-temperature range $(<250 \mathrm{~K})$, their thermal conductivity is relatively high $\left(\sim 4-8 \mathrm{~W} \mathrm{~m}^{-1} \mathrm{~K}^{-1}\right)$, and the materials need to be compositionally tuned further to make them useful as thermoelectrics.

Recently, cubic quaternary compounds with a complex formula $\mathrm{Ag}_{n} \mathrm{~Pb}_{m} \mathrm{M}_{n} \mathrm{Te}_{m+2 n}$ $(\mathrm{M}=\mathrm{Sb}, \mathrm{Bi})$, crystallizing in the PbTe structure, have been reported. ${ }^{23}$ The composition $\mathrm{AgPb}_{10} \mathrm{SbTe}_{12}$ shows an exceptionally high $Z T$ value $(>2)$ at elevated temperature (shown in Figure 2). This is due to the very low total thermal conductivity of the bulk material, possibly arising from compositional modulations (seen as "nanodots") similar to the one found in superlattices. If this is verified, it provides an additional "knob" to turn to achieve high ZT in bulk materials. Another group of materials under investigation are half-Heusler alloys, with the general formula MNiSn ( $\mathrm{M}=\mathrm{Zr}$, Hf, Ti). A complex composition of the type $\mathrm{Zr}_{0.5} \mathrm{Hf}_{0.5} \mathrm{Ni}_{0.5} \mathrm{Pd}_{0.5} \mathrm{Sn}_{0.99} \mathrm{Sb}_{0.01}$ shows a $\mathrm{ZT}$ of 0.7 at $T \approx 800 \mathrm{~K}$, highlighting the intricate balance in structure, composition, and property relationships in these compounds. ${ }^{24}$ The $\beta-\mathrm{Zn}_{4} \mathrm{Sb}_{3}$ system has been reinvestigated for TE power-generation applications at the Jet Propulsion Laboratory. ${ }^{25}$

Crystal Structures with "Rattlers." The method of lowering the lattice thermal conductivity through mixed-crystal or solid-solution formation does not always produce enough phonon scattering to lower the lattice thermal conductivity to $\kappa_{\min }$. Slack's concept of a "phononglass/electron-crystal," described earlier, avoids this limitation. The concept of $\kappa_{\text {min }}$ is successfully verified in crystal structures with large empty cages or voids where atoms can be partially or completely filled in such a way that they "rattle," resulting in the scattering of the acoustic phonons. This approach especially works well in highly covalent semiconductor materials based on clathrates ( $\mathrm{Si}, \mathrm{Ge}$, or $\mathrm{Sn}$ ) and void structures formed by heavy elements of low electronegativity differences (e.g., $\mathrm{CoSb}_{3}$-based skutterudites). Some doped skutterudites show exceptionally high $Z T$ values at elevated temperatures $(Z T \approx 1.5$ at $600-800 \mathrm{~K}$ ). The structure-property relationships of these materials are discussed in the article by Nolas et al. in this issue.

Oxide Thermoelectrics. There are numerous oxides with metal atoms in their common oxidation states that are stable at elevated temperatures and show electrical properties ranging from insulating to superconducting. Nevertheless, oxides have received very little attention for TE applications. This is due to their strong ionic character, with narrow conduction bandwidths arising from weak orbital overlap, leading to localized electrons with low carrier mobilities. This situation changed with the unexpected discovery of good TE properties in a strongly correlated layered oxide, $\mathrm{NaCO}_{2} \mathrm{O}_{4} \cdot{ }^{26}$ This oxide attains $\mathrm{ZT} \approx$ $0.7-0.8$ at $1000 \mathrm{~K}$. Inspired by the striking TE performance of $\mathrm{NaCO}_{2} \mathrm{O}_{4}$, most of the current studies are focused on Co-based layered oxides, such as $\mathrm{Ca}_{3} \mathrm{Co}_{4} \mathrm{O}_{9}$ and $\mathrm{Bi}_{2} \mathrm{Sr}_{3} \mathrm{Co}_{2} \mathrm{O}_{y}$, crystallizing in "misfit" (lattice-mismatched) layered structures. ${ }^{26}$ Among the $n$-type oxides, Al-doped $\mathrm{ZnO}$ $\left(\mathrm{Al}_{0.02} \mathrm{Zn}_{0.98} \mathrm{O}\right)$ shows reasonably good $\mathrm{TE}$ performance $(Z T \approx 0.3$ at $1000 \mathrm{~K}){ }^{27}$

Rare-Earth Intermetallics with High Power Factors. As mentioned earlier, metallic compounds are not suitable for TE applications. The exceptions to this rule are intermetallic compounds containing rare-earth elements (e.g., $\mathrm{Ce}$ and $\mathrm{Yb}$ ), with localized magnetic moments where the Seebeck coefficient can approach $\sim 100 \mu \mathrm{V} / \mathrm{K}$ with metal-like conductivities. ${ }^{28,29}$ In these compounds, the $4 f$ levels lie near the Fermi energy and form narrow non-parabolic bands, resulting in a large density of states at the Fermi level and large Seebeck coefficient values. The highest Seebeck values are found in cubic $\mathrm{YbAl}_{3}$ (n-type) and $\mathrm{CePd}_{3}$ (p-type). $\mathrm{YbAl}_{3}$ shows a very high power factor (120-180 $\mu \mathrm{W} / \mathrm{cm} \mathrm{K}^{2}$, or 3.6-5.4 W m ${ }^{-1} \mathrm{~K}^{-1}$ ) at room temperature $(300 \mathrm{~K})$, which is nearly 4-5 times larger than that observed in optimized $\mathrm{Bi}_{2} \mathrm{Te}_{3}$-based thermoelectrics. ${ }^{30}$ Unfortunately, the large thermal conductivity (15-22 W m $\left.\mathrm{m}^{-1} \mathrm{~K}^{-1}\right)$ lowers the ZT to about 0.2 at room temperature. Recently, the lattice thermal conductivity of this system has been lowered by doping $\mathrm{Mn}$ in the interstitial positions, resulting in the increase of ZT to about 0.4 at room temperature. ${ }^{31}$ As mentioned earlier, $Z T=1$ requires a minimum Seebeck coefficient value of $156 \mu \mathrm{V} / \mathrm{K}$. The correlated metal with the highest Seebeck coefficient is $\mathrm{CePd}_{3}$, which has a maximum of $125 \mu \mathrm{V} / \mathrm{K}$ at $140 \mathrm{~K} .{ }^{29}$ Future investigations should focus on increasing the Seebeck coefficients of these materials above $\sim 150 \mu \mathrm{V} / \mathrm{K}$ through compositional and structural tuning.

Engineered Crystal Lattices. The approaches in bulk materials research rely heavily on the thermodynamic stability of the phases at a given condition, whereas thin-film deposition can yield metastable "designer" phases with unique properties. Quantum well systems (0D, 1D, and 2D) take advantage of their low-dimensional character through physical confinements in quantum dots, nanowires, and thin-film structures to enhance the electronic properties of a given material. ${ }^{32}$ In addition, nanostructured semiconductor materials could scatter mid- to long-wavelength phonons and thereby reduce the lattice thermal conductivity to $\kappa_{\min }$.

Researchers at the Research Triangle Institute (RTI) have demonstrated a significant enhancement in $\mathrm{ZT}$ through the construction of $\mathrm{Bi}_{2} \mathrm{Te}_{3} / \mathrm{Sb}_{2} \mathrm{Te}_{3}$ superlattices. $^{33}$ These materials exhibited $\mathrm{ZT} \approx 2.4$ at $T \approx 330 \mathrm{~K}$. The enhancement is attributed to creating a "nanoengineered" material that is efficient in thermal insulation while remaining a good electrical conductor. The thermal insulation arises from a complex localized behavior for phonons, while the electron transmission is facilitated by optimal choice of band offsets in these semiconductor heterostructures. Also, there have been reports on $\mathrm{PbTe} / \mathrm{PbTeSe}$ quantum dot structures that yield $Z T \approx 1.3-1.6 .{ }^{34}$ These materials have been grown as thick films that are then "floated off" the substrate to yield freestanding films, which were measured to yield these results. The enhancement in ZT in the superlattice materials appears to be more from a reduction in the lattice thermal conductivity than an increase in power factor.

\section{Summary}

Currently, there are no theoretical or thermodynamic limits to the possible 
values of ZT. Given the current need for alternative energy technologies and materials to replace the shrinking supply of fossil fuels, the effort is becoming more urgent. Energy-related research will grow rapidly over the next few years, and higher-performance thermoelectric materials and devices are direly needed. Slack estimated that an optimized phononglass/electron-crystal material could possibly exhibit values of $Z T \approx 4 .{ }^{14}$ This gives encouragement that such materials may be possible and could address many of our energy-related problems. Thus, a systematic search and subsequent thorough investigation may eventually yield these much-needed materials for the next generation of TE devices.

Although many strategies are being employed in hopes of identifying novel TE materials, the PGEC approach appears to be the best, as will become apparent in the following articles. One has to decide whether "holey" semiconductors (materials with cages, such as skutterudites or clathrates) or "unholey" semiconductors (such as $\mathrm{SiGe}$ or $\mathrm{PbTe}$ ) are the best to pursue, and which tuning parameters are available to improve these materials..$^{35}$ To date, none of the new materials has displaced the current state-of-the-art materials $\left(\mathrm{Bi}_{2} \mathrm{Te}_{3}, \mathrm{PbTe}\right.$, or $\left.\mathrm{SiGe}\right)$ in a commercial TE device. These materials have held that distinction for more than 30 years.

However, given the many materials yet to be investigated, there is certainly much more work ahead and promise for developing higher-efficiency thermoelectric materials and devices. While the results are very exciting, thin films may be most appropriate for small-scale electronic and optoelectronics applications where small heat loads or low levels of power generation are more appropriate. To address large-scale refrigeration (home refrigerators) or power-generation (automotive or industrial) requirements, higher-performance bulk materials will have to be developed.

Certainly, theoretical guidance, in terms of band structure calculations and modeling, will be essential to identifying the most promising TE materials. In addition, rapid yet accurate characterization of materials and verification of results are also essential in order to effectively advance this field of research. A multidisciplinary approach will be required to develop higher-efficiency thermoelectric materials and devices. The techniques used to develop "designer materials" needed for thermoelectrics will most likely prove important in other areas of materials research as well.

\section{References}

1. The reader is referred to the many MRS Symposium Proceedings volumes on the topic of thermoelectric materials and energyconversion technologies: Thermoelectric Materials-New Directions and Approaches (Mater. Res. Soc. Symp. Proc. 478, 1997); Thermoelectric Materials 1998-The Next Generation Materials for Small-Scale Refrigeration and Power Generation Applications (Mater. Res. Soc. Symp. Proc. 545, 1999); Thermoelectric Materials 2000-The Next Generation Materials for Small-Scale Refrigeration and Power Generation Applications (Mater. Res. Soc. Symp. Proc. 626, 2001); Thermoelectric Materials 2001-Research and Applications (Mater. Res. Soc. Symp. Proc. 691, 2002); Thermoelectric Materials 2003-Research and Applications (Mater. Res. Soc. Symp. Proc. 793, 2004); and Materials and Technologies for Direct Thermal-toElectric Energy Conversion (Mater. Res. Soc. Symp. Proc. 886, 2006) in press.

2. T.M. Tritt, ed., "Recent Trends in Thermoelectric Materials Research," Semiconductors and Semimetals, Vols. 69-71, treatise editors, R.K. Willardson and E. Weber (Academic Press, New York, 2000).

3. Jet Propulsion Laboratory Thermoelectric Science and Engineering Web site, http:/ / www.its.caltech.edu/ jsnyder/thermoelectrics/ (accessed February 2006).

4. A.W. Allen, Laser Focus World 33 (March 1997) p. S15.

5. G.S. Nolas, J. Sharp, and H.J. Goldsmid, Thermoelectrics: Basic Principles and New Materials Developments (Springer, New York, 2001).

6. T.J. Seebeck, Abh. K. Akad. Wiss. (Berlin, 1823) p. 265.

7. D.T. Morelli, in Encyclopedia of Applied Physics, Vol. 21 (1997) p. 339.

8. P.M. Chaiken, in Organic Superconductors, edited by V.Z. Kresin and W.A. Little (Plenum Press, New York, 1990) p. 101.

9. A.F. Ioffe, Semiconductor Thermoelements and Thermoelectric Cooling (Infosearch, London, 1957).

10. J.C. Peltier, Ann. Chem. LVI (1834) p. 371.

11. H.J. Goldsmid, Electronic Refrigeration (Pion Limited, London, 1986).

12. D.M. Rowe, ed., CRC Handbook of Thermoelectrics (CRC Press, Boca Raton, FL, 1995).

13. P.L. Rossiter, The Electrical Resistivity of Metals $\mathcal{E}$ Alloys (Cambridge Press, New York, 1987); F.J. Blatt, Physics of Electronic Conduction in Solids (McGraw-Hill, New York, 1968); L. Solymar and D. Walsh, Electrical Properties of Materials, 6th Ed. (Oxford Press, New York, 1998).

14. G.A. Slack, in CRC Handbook of Thermoelectrics, ed. by D.M. Rowe (CRC Press, Boca Raton, FL, 1995) p. 407.

15. G.D. Mahan and J.O. Sofo, Proc. Natl. Acad. Sci. USA 93 (1996) p. 7436.

16. G.D. Mahan, B. Sales, and J. Sharp, Phys. Today 50 (3) (1997) p. 42.

17. G.A. Slack, in Solid State Physics, Vol. 34, edited by F. Seitz, D. Turnbull, and H. Ehrenreich (Academic Press, New York, 1979) p. 1.

18. R.R. Heikes and R.W. Ure Jr., Thermoelectricity: Science and Engineering (Wiley Interscience, New York, 1961) p. 405.

19. C. Wood, Rep. Prog. Phys. 51 (1988) p. 459. 20. M.G. Kanatzidis, S.D. Mahanti, and T.P. Hogan, eds., Chemistry, Physics and Materials Science of Thermoelectric Materials: Beyond Bismuth Telluride (Plenum, New York, 2003) p. 35.

21. D.Y. Chung, T. Hogan, P. Brazis, M. Rocci-Lane, C. Kannewurf, M. Bastea, C. Uher, and M.G. Kanatzidis, Science 287 (2000) p. 1024.

22. R.T. Littleton IV, T.M. Tritt, M. Korzenski, D. Ketchum, and J.W. Kolis, Phys. Rev. B. Rap. Commun. 64121104 (2001).

23. K.F. Hsu, S. Loo, F. Gao, W. Chen, J.S. Dyck, C. Uher, T. Hogan, E.K. Polychroniadis, and M. Kanatzidis, Science 303 (2004) p. 8181.

24. Q. Shen, L. Chen, T. Goto, T. Hirai, J. Yang, G.P. Meisner, and C. Uher, Appl. Phys. Lett. 79 (2001) p. 4165.

25. T. Caillat, J.P. Fleurial, and A. Borshchevsky, J. Phys. Chem. Solids 58 (1997) p. 1119.

26. I. Terasaki and N. Murayama, eds., Oxide Thermoelectrics (Research Signpost, Trivandrum, India, 2002).

27. M. Ohtaki, T. Tsubota, K. Eguchi, and H. Arai, J. Appl. Phys. 79 (1996) p. 1816.

28. R.J. Gambino, W.D. Grobman, and A.M. Toxen, Appl. Phys. Lett. 22 (1973) p. 506.

29. G.D. Mahan, in Solid State Physics, edited by F. Seitz, H. Ehrenreich, and F. Spaepen (Academic Press, New York, 1997) p. 51.

30. D.M. Rowe, G. Min, and V.L. Kuznetsov, Philos. Mag. Lett. 77 (1998) p. 105; D.M. Rowe, V.L. Kuznetsov, L.A. Kuznetsova, and G. Min, J. Phys. D: Appl. Phys. 35 (2002) p. 2183.

31. T. He, T.G. Calvarese, J.-Z. Chen, H.D. Rosenfeld, R.J. Small, J.J. Krajewski, and M.A. Subramanian, Proc. 24th Int. Conf. on Thermoelectrics, edited by T.M. Tritt (IEEE, Piscataway, NJ, 2005) p. 434.

32. L.D. Hicks and M.S. Dresselhaus, Phys. Rev. B 47 (1993) p. 12727.

33. R. Venkatasubramanian, E. Siivola, T. Colpitts, and B. O'Quinn, Nature 13 (2001) p. 597.

34. T.C. Harman, P.J. Taylor, M.P. Walsh, and B.E. LaForge, Science 297 (2002) p. 2229. 35. T.M. Tritt, Science 283 (1999) p. 804.

\section{Missing Important Issues of MRS Bulletin?} Back Issues are still available! Contact MRS for details- 
Terry M. Tritt, Guest Editor for this issue of MRS Bulletin, is a professor of physics at Clemson University. He received both his $\mathrm{BA}$ degree (1980) and his $\mathrm{PhD}$ degree (1985) in physics from Clemson University and then went to the Naval Research Laboratory (NRL) under a National Research Council postdoctoral fellowship. He subsequently became a staff scientist at NRL, where he remained for 11 years before joining the faculty at Clemson in 1996. His primary research expertise lies in electrical and thermal transport properties and phenomena, and especially in measurement and characterization techniques in novel materials. He has extensive expertise in thermoelectric materials and measurement science and has built an internationally known laboratory for the measurement and characterization of thermoelectric material parameters, particularly thermal conductivity. $\mathrm{He}$ has recently become involved in the synthesis and characterization of thermoelectric nanomaterials.

Tritt has served as lead organizer of three Materials Research Society symposia on thermoelectric materials, edited the three-volume Recent Trends in Thermoelectric Materials Research (Academic Press, 2000), and also recently edited a book published by Kluwer Press on thermal conductivity. He has been a member of the executive board of the International Thermoelectric Society (ITS) since 1999 and served as chair and host of the 24th International Conference on Thermoelectrics (ICT-2005)

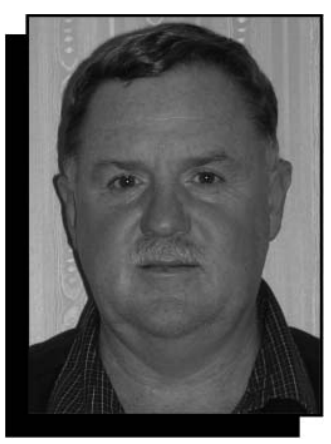

Terry M. Tritt

at Clemson in June 2005. He has written more than 140 journal publications and regularly gives invited presentations at national and international meetings.

Tritt can be reached at Clemson University, Department of Physics, 103 Kinard Laboratory, Clemson, SC 29634, USA; tel. 864-656-5319 and e-mail ttritt@ clemson.edu.

\section{M.A. Subramanian,} Guest Editor for this issue of MRS Bulletin, is a research fellow at DuPont Central Research and Development. He holds BS and MS degrees in chemistry from the University of Madras in India and received his $\mathrm{PhD}$ degree in solid-state chemistry in 1982 from the Indian Institute of Technology in Madras, where he focused on synthesis and solid-state studies of oxides with pyrochlore and perovskite structures. He subsequently joined the Department of Chemistry at Texas A\&M University as an NSF postdoctoral fellow, where he worked on designing new fast ion conductors for solidstate batteries. He joined DuPont in 1985 as a scientist and was recently appointed to research fellow. Subramanian's current interests include

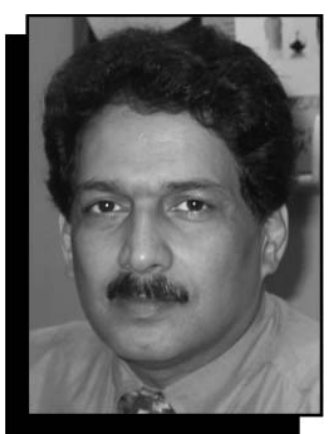

M.A. Subramanian

the design and understanding of structureproperty relationships in new solid-state inorganic functional materials related to superconductivity, colossal magnetoresistive materials, high- $\kappa$ and low- $\kappa$ dielectrics, ferroelectrics, multiferroics, oxyfluorination, and thermoelectrics.

Subramanian is a visiting professor at the Institut de Chimie de la Matière Condensée de Bordeaux (ICMCB), University of Bordeaux, France. He serves as editor for Solid State Sciences and Progress in Solid State Chemistry, and serves or has served on the editorial boards of the Materials Research Bulletin, Chemistry of Ma terials, and the Journal of Materials Chemistry. $\mathrm{He}$ was awarded the Charles Pedersen Medal by DuPont in 2004 for his outstanding scientific, technological, and business contributions to the company. He has authored more than 200 publications and holds 42 U.S. patents, with 10 applications pending.

Subramanian can be reached at DuPont Central Research and Development, Experimental Station, E328/219, Wilmington, DE 198800328, USA; tel. 302-6952966 and e-mail mas.subramanian@usa. dupont.com.

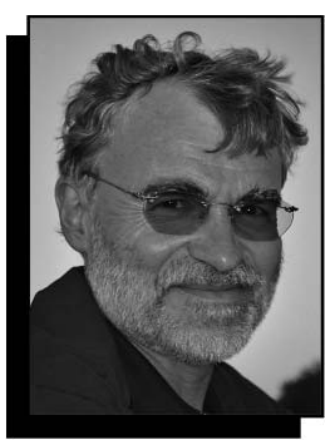

Harald Böttner

Harald Böttner is deputy head of the Components and Microsystems Department of the Fraunhofer Institute for Physical Measurement Techniques in Freiburg, Germany, and is currently responsible for its thermoelectric activities. He graduated with a diploma degree in chemistry from the University of Münster in 1974 and received his $\mathrm{PhD}$ degree in 1977 at the same university for his thesis on diffusion and solid-state reaction in the quaternary semiconductor II-VI/IV-VI material system. He joined the Fraunhofer Institut für Silicatforschung in 1978 and accepted his present appointment in $1980 . \mathrm{He}$ developed IV-VI infrared semiconductor lasers in parallel with activities in thermoelectrics until 1995, when he became responsible for the development of semiconductor gas sensors and thermoelectric materials until 2003. Böttner's current research activities focus on thin-film and nanoscale thermoelectrics and microelectronics-related device technology. He is the author or co-author of more than 100 publications, holds more than 10 patents, and is a member of the board

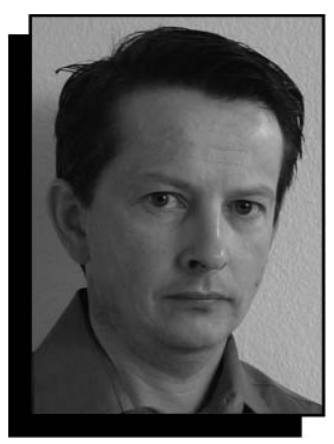

Thierry Caillat

of the International Thermoelectric Society.

Böttner can be reached at Fraunhofer IPM, Research Field Thermoelectrics, Heidenhofstrasse 8, 79110 Freiburg, Germany; tel. 49-761-8857121 and e-mail harald.boettner@ ipm.fraunhofer.de.

Thierry Caillat is a senior member of technical staff with the Jet Propulsion Laboratory at the California Institute of Technology. He received his $\mathrm{PhD}$ degree in materials science from the National Polytechnique Institute of Lorraine, France, in 1991. He then received a National Research Council fellowship to study new materials for thermoelectric applications at JPL. He joined the permanent staff at JPL in 1994. Caillat's primary research interests have focused on the identification and development of new thermoelectric materials and devices. In the last ten years, he has played a key role in identifying several families of promising compounds for thermoelectric applications, including skutterudites and $\beta-\mathrm{Zn}_{4} \mathrm{Sb}_{3}$-based materials. More recently, he has been involved in the development of advanced thermoelectric power-generation devices for both 
space and terrestrial applications.

Caillat has authored or co-authored more than 100 publications, given more than 40 invited presentations, and served on numerous national and international organization committees and panels. He was also a board member of the International Thermoelectric Society from 1996 to 2005.

Caillat can be reached at the California Institute of Technology, Jet Propulsion Laboratory, Mail Stop 277/207, 4800 Oak Grove Drive, Pasadena, CA 91109, USA; tel. 818-354-0407 and e-mail thierry. caillat@jpl.nasa.gov.

Gang Chen is a professor of mechanical engineering at the Massachusetts Institute of Technology. He received his BS (1984) and MS (1987) degrees from the Power Engineering Department at

Huazhong University of Science and Technology, China, in 1984 and 1987, respectively, and his PhD degree in mechanical engineering from the University of California, Berkeley, in 1993. He was an assistant professor at Duke University from 1993 to 1997 and an associate professor at the University of California, Los Angeles, from 1997 to 2001 . His research interests center on microelectronics thermal management and nanoscale transport phenomena, particularly nanoscale heat transfer, and their applications in energy storage and conversion.

Chen is a recipient of a K.C. Wong Education Foundation fellowship, an NSF Young Investigator Award, and a John Simon Guggenheim

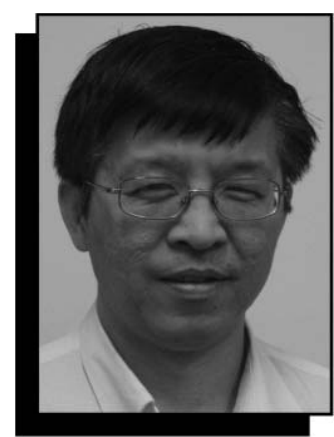

Gang Chen

Foundation fellowship. He serves on the editorial boards of Annual Review of Heat Transfer, the Journal of Computational and Theoretical

Nanoscience, the Journal of Nanomaterials, and Microscale Thermophysical Engineering. He also serves as the chair of the Advisory Board of the ASME Nanotechnology Institute and on the advisory boards of several organizations.

Chen can be reached at the Massachusetts Institute of Technology, Department of Mechanical Engineering, Room 3-158, 77 Massachusetts Avenue, Cambridge, MA 02139-4307, USA; tel. 617-253-0006 and e-mail gchen2@ mit.edu.

Ryoji Funahashi has been a senior researcher at the National Institute of Advanced Industrial Science and Technology in Japan since 1992. He received his BS (1989) and MS (1992) degrees in physical chemistry and his $\mathrm{PhD}$ degree (1998) in crystalline material science from Nagoya University. His research interests include novel synthetic techniques for highperformance superconducting oxide materials and, recently, the exploration of new thermoelectric oxide materials.

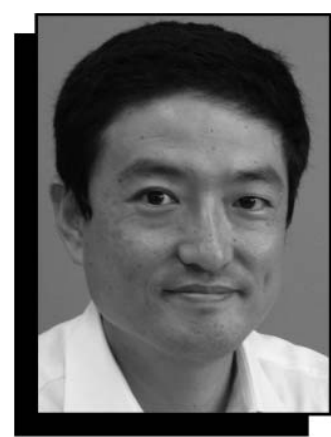

Ryoji Funahashi

Funahashi is a recipient of the Thermoelectric Conference of Japan's Best Paper Award, the Japan Journal of Applied Physics Paper Award, and a NEDO Industrial Technology Research and Development Project grant. He has authored or coauthored more than 150 papers, conference proceedings, invited talks, and reviews. He is also a board member of the International Thermoelectric Society and the Thermoelectric

Society of Japan.

Funahashi can be reached at AIST, UBIQEN, Molecular Materials and Devices, 1-8-31 Midorigaoka, Ikeda, Osaka 563-8577, Japan; tel. 81-727-519485 and e-mail funahashi-r@aist.go.jp.

Xiaohua Ji is a postdoctoral researcher in the Department of Physics and Astronomy at Clemson University. In 2005, she received her $\mathrm{PhD}$ degree in materials physics and chemistry from Zhejiang University in China under the guidance of Xinbing Zhao. For her graduate work, she developed solvothermal and hydrothermal methods for synthesizing nanostructured thermoelectric materials. She received the Best Scientific Paper Award at the

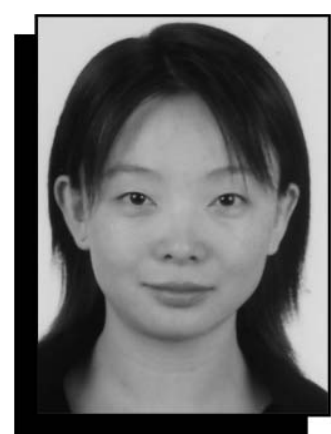

Xiaohua Ji

2004 ICT meeting in Adelaide, Australia. Under the guidance of Terry M. Tritt at Clemson, her current research involves the synthesis and characterization of nanostructured thermoelectric materials and nanocomposite bulk thermoelectric materials.

Ji can be reached at Clemson University, Department of Physics and Astronomy, Clemson, SC 29634, USA; tel. 864656-4596 and e-mail xiaohji@clemson.edu.

Mercouri Kanatzidis is a University Distinguished Professor of Chemistry at Michigan State University, where he has served on the faculty since 1987. He received his BS degree from Aristotle University in Greece, followed by a PhD degree in chemistry from the University of Iowa in 1984. He was then a postdoctoral research associate at Northwestern University from 1985 to 1987. He has generated seminal work in metal chalcogenide chemistry through the development of novel synthetic approaches aimed at new materials discovery. His research interests include novel chalcogenides, thermoelectric materials, and the design of framework solids, intermetallic phases, and organic-inorganic nanocomposites.

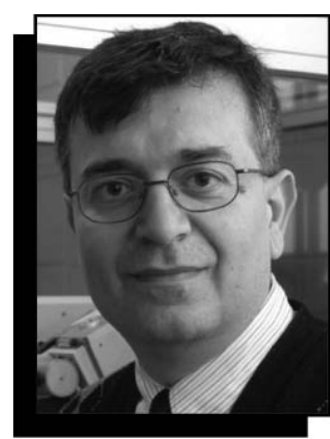

Mercouri Kanatzidis

Kanatzidis is a recipient of the Presidential Young Investigator Award, the ACS Morley Medal, the ACS Exxon Solid State Chemistry Award, and the Humboldt Prize. He has been a Guggenheim fellow as well as a visiting professor at the University of Nantes, the University of Münster, and the University of Munich. The bulk of his work is described in more than 450 research publications. He holds six patents and is editor in chief of the Journal of Solid State Chemistry.

Kanatzidis can be reached at Michigan State University, Department of Chemistry, 406 Chemistry Building, East Lansing, MI 488241322, USA; tel. 517-355-

9715 and e-mail

kanatzid@cem.msu.edu.

Kunihito Koumoto is a professor in the Graduate School of Engineering at Nagoya

University, Japan. He received BS, MS, and $\mathrm{PhD}$ degrees in applied chemistry from the University of Tokyo in 1974, 1976, and 1979, respectively. He served as an assistant professor and associate professor at the University of Tokyo before joining Nagoya University as a full professor in 1992. His research focuses on thermoelectric materials 


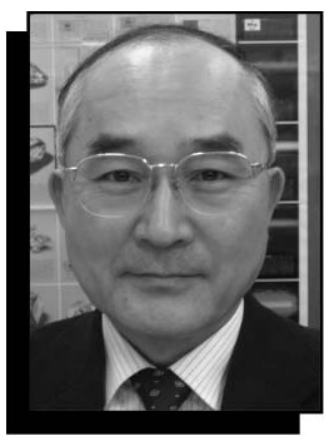

Kunihito Koumoto

and bio-inspired processing of inorganic materials.

Koumoto received the Richard M. Fulrath Award in 1993 and the Academic Achievement Award of the Ceramic Society of Japan in 2000. He became a fellow of the American Ceramic Society and received the Chinese Ceramic Society Award in 2005. He also served the International Thermoelectric Society as president from 2003 to 2005 and is the author or co-author of more than 320 scientific papers and 38 books.

Koumoto can be reached at Nagoya University, Graduate School of Engineering, Furo-cho, Chikusa-ku, Nagoya, 464-8603, Japan; tel. 81-52-7893327 and e-mail koumoto@apchem. nagoya-u.ac.jp.

George S. Nolas is an associate professor of physics at the University of South Florida, where he has been since 2001. He received his PhD degree from Stevens Institute of Technology in 1994 and conducted pioneering studies on the synthesis and thermal properties of filled skutterudites as a postdoctoral associate with Glen Slack at Rensselaer Polytechnic Institute. Before accepting his current position,

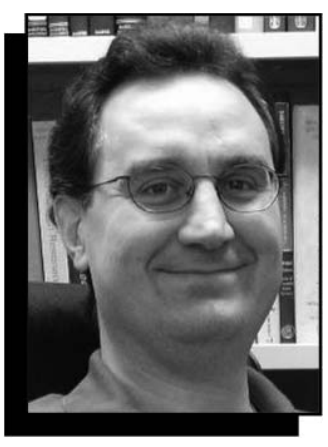

George S. Nolas

he spent five years as a physicist and senior member of the technical staff at Marlow Industries Inc., a thermoelectrics manufacturer in Dallas, Texas. His research interests are in the synthesis and structure-property relationships of new materials, and his current research focuses on new materials for power conversion and alternative energy applications, including thermoelectrics, photovoltaics, and hydrogen storage.

Nolas is author of Thermoelectrics: Basic Principles and New Materials Developments, published by Springer with co-authors Jeffrey Sharp and Julian Goldsmid. He holds three patents, with another two pending, on new materials for power-conversion applications. He has edited four Materials Research Society proceedings volumes (two as lead editor) on thermoelectric materials research, organized symposia for the American Physical Society and the American Ceramic Society, given numerous invited conference presentations and seminars, and is currently in his second term as a board member of the International Thermoelectric Society.

Nolas can be reached at the University of South Florida, Depart-

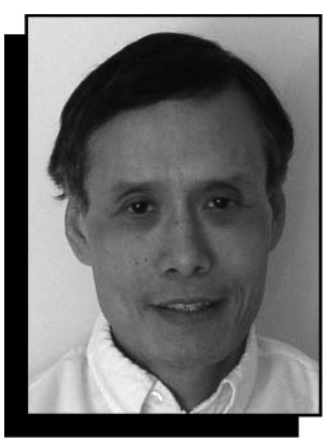

Joseph Poon

ment of Physics, PHY 114, 4202 E. Fowler Avenue, Tampa, FL 336205700, USA; tel. 813-974-2233 and e-mail gnolas@cas.usf.edu.

Joseph Poon is the William Barton Rogers Professor of Physics at the University of Virginia, where he joined the Physics Department in 1980. He received both his $\mathrm{BS}$ and $\mathrm{PhD}$ degrees from the California Institute of Technology and was a research associate in applied physics at Stanford University. His areas of research have included amorphous superconductors, quasicrystals, bulk amorphous metals, and thermoelectric alloys. He is a fellow of the American Physical Society and was named one of the "Scientific American 50" in 2004 for the creation of amorphous steel. He has published more than 200 papers.

Poon can be reached at the University of Virginia, Department of Astronomy-Physics, PO Box 400714, Jesse Beams Lab, Room 167,

Charlottesville, VA

22901, USA; tel. 434-924-

6792 and e-mail

sjp9x@virginia.edu.

Apparao M. Rao is a professor in condensedmatter physics at Clemson University. He

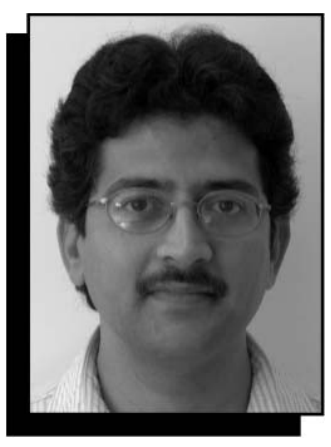

Apparao M. Rao

obtained his $\mathrm{PhD}$ degree in physics from the University of Kentucky in 1989 and held a postdoctoral appointment with Mildred S. Dresselhaus at MIT until 1991. His current research focuses on understanding and controlling the synthesis of 1D nanostructured organic and inorganic materials. He has published extensively on the synthesis, characterization, and applications of carbon nanotubes.

Rao can be reached at Clemson University, Department of Physics and Astronomy, 107 Kinard Laboratory, Clemson, SC 29634-0978, USA; tel. 864-656-6758 and e-mail arao@clemson.edu.

Ichiro Terasaki is a professor in the Applied Physics Department at Waseda University in Tokyo. He received his $\mathrm{BE}, \mathrm{ME}$, and $\mathrm{PhD}$ degrees in applied physics from the University of Tokyo in 1986, 1988, and 1992, respectively. He began his research career as a research associate at the University of Tokyo, moving on to become chief researcher at the International Superconductivity Technology Center. He became an associate professor at Waseda in 1997 and accepted his current position as a full professor in 2004. His research in- terests include experimental studies in condensed-matter physics, especially charge transport properties of transition-metal oxides, organic conductors, and intermetallic compounds.

Terasaki is the recipient of the Sir Martin Wood Prize and ICT's Best Scientific Paper Award. He has published more than 150 papers, conference proceedings, invited talks, books, and reviews.

Terasaki can be reached at Waseda University, Department of Applied Physics, 3-4-1 Okubo, Shinjuku-ku, Tokyo 169-8555, Japan; tel. 81-3-5286-3854 and e-mail terra@waseda.jp.

Rama Venkatasubramanian is director of the Center for Thermoelectric Research at RTI International in North Carolina and the founder and chief technology officer of Nextreme Thermal Solutions Inc., a company spun off from RTI to commercialize its unique thin-film superlattice thermoelectric technology. He earned his PhD degree in electrical engineering from Rensselaer Polytechnic Institute and is a National Talent Scholar and a graduate of the Indian Institute of Technology in Madras. His research interests include photovoltaics, heteroepitaxy of novel materials, photonic materials, the study of nanoscale thermal physics, thermal management in highperformance electronics, and direct thermal-toelectric conversion devices. He is well known for pioneering thermoelectric superlattice materials and devices. Venkatasubramanian's 


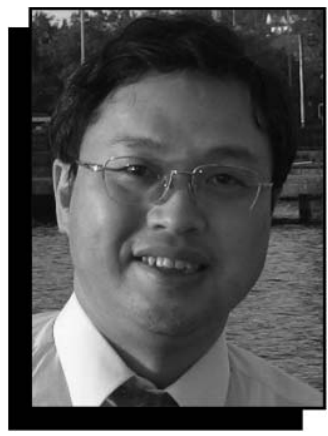

Ichiro Terasaki

work on superlattices has been recognized as a significant breakthrough in thermoelectrics using nanoscale engineered materials. This technology has won an R\&D 100 Award (2002) and the Technology of the Year Award (2005) from the Council for Entrepreneurial Development in North Carolina.

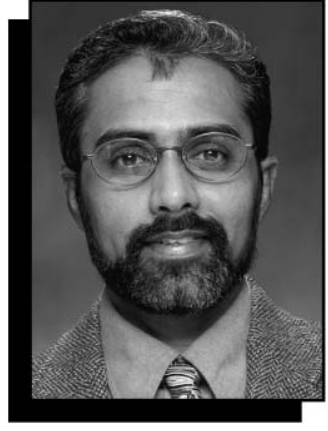

Rama

Venkatasubramanian

Venkatasubramanian is a recipient of the Allen B. Dumont Prize from Rensselaer, RTI's Margaret Knox Excellence Award in Research in 2002, and the IEEE Eastern North Carolina Inventor of the Year in 2003. He has several patents issued in thermoelectrics, has authored more than 100 refereed publications,

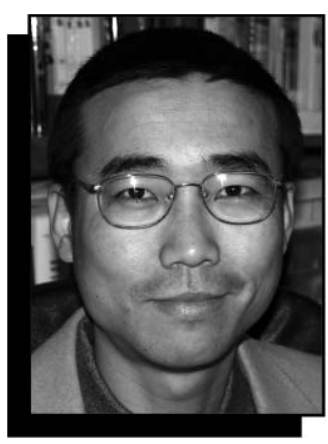

Jihui Yang and has contributed to two book chapters.

Venkatasubramanian can be reached at RTI International, Center for Thermoelectric

Research, PO Box 12194, Research Triangle Park, NC 27709-2194, USA; tel. 919-541-6889 and e-mail rama@rti.org.

Jihui Yang is a staff research scientist in the
Materials and Processes Laboratory at General Motors Research and Development Center. He received a BS degree in physics from Fudan University of China in 1989, an MS degree in physics from the University of Oregon in 1991, an MS degree in radiological physics from Wayne State University in 1994, and a $\mathrm{PhD}$ degree in physics from the University of Michigan in 2000. His research interests include low-temperature transport properties of intermetallic compounds and semiconductors, magnetism, thermoelectric materials, and the development of thermoelectric technology for automotive applications.

Yang has published several book chapters and more than 30 papers. He was the recipient of the GM DEGS fellowship in 1997 and the Kent M. Terwilliger Prize for Best Doctoral Thesis from the Physics Department of the University of Michigan in 2001. He has served on various committees for APS and the Materials Research Society and organized several symposia for MRS and ACerS. He was also elected to the board of directors of the International Thermoelectric Society in 2005.

Yang can be reached at General Motors Research and Development Center, Mail Code 480-106-224, 30500 Mound Road, Warren, MI 48090, USA; tel. 586986-9789 and e-mail jihui.yang@gm.com.

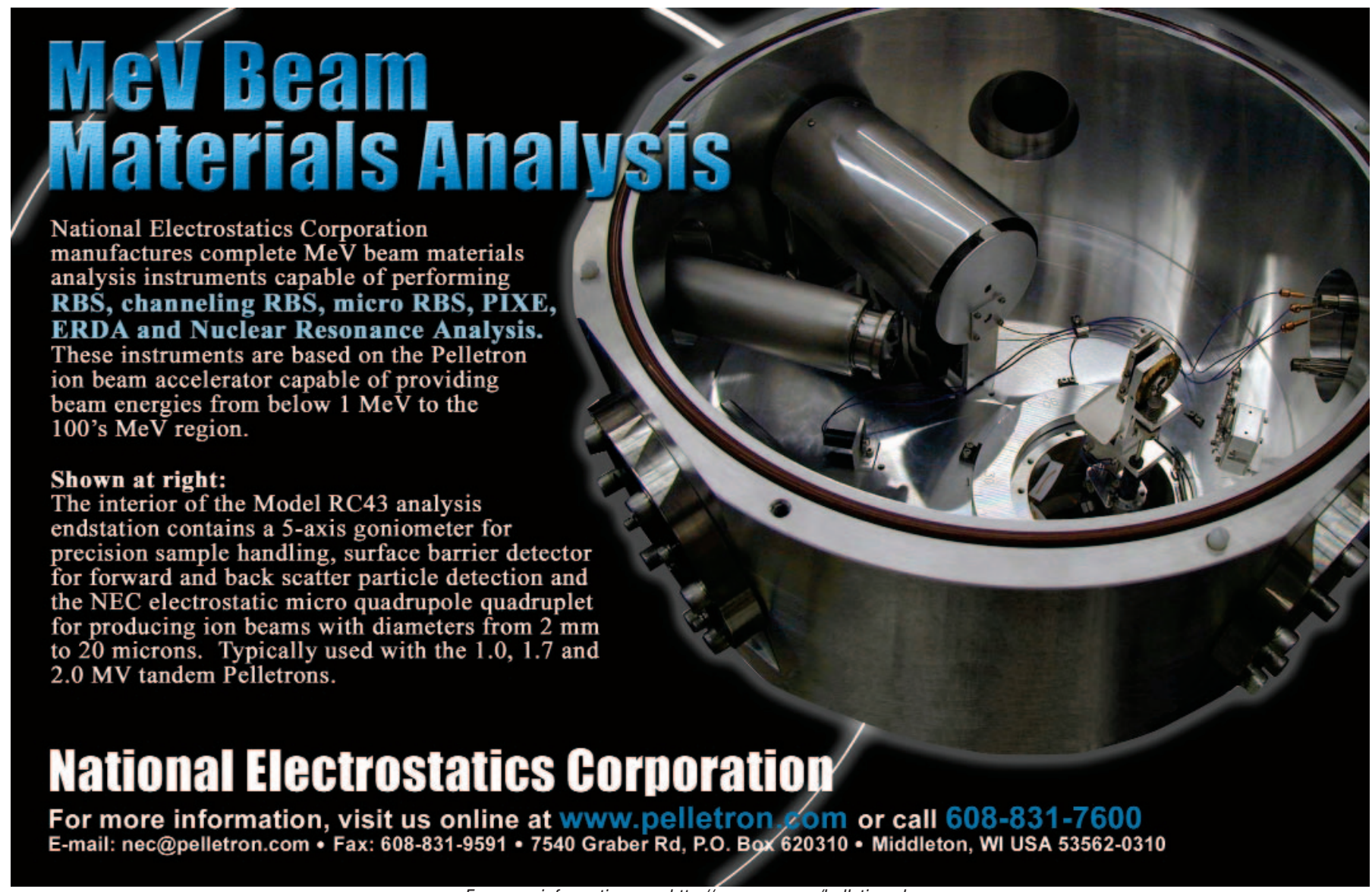

For more information, see http://WWW.mrs.org/bulletin_ads 